\title{
En el sangrado gastrointestinal alto agudo es mejor una estrategia transfusional restrictiva
}

\author{
A restrictive transfusion strategy is better in acute upper gastrointestinal bleeding
}

Villanueva c. y col. N Engl J Med 2013; 368:11-21.

\section{Objetivos}

Comparar la efectividad y seguridad de una estrategia de transfusión restrictiva contra una estrategia liberal en el sangrado gastrointestinal alto agudo (SGAA).

\section{Diseño lugar y pacientes}

Ensayo clínico aleatorizado, controlado, realizado en Barcelona (junio 2003 a diciembre 2009). Se evaluaron pacientes internados por SGAA no masivo. De 1610 pacientes, 648 fueron excluidos, 41 se negaron y 32 abandonaron tras la aleatorización. Se incluyeron 889 pacientes para el análisis. La aleatorización se estratificó según la presencia o ausencia de cirrosis.

\section{Intervención}

Se realizó endoscopia digestiva alta a todos los pacientes dentro de las primeras seis horas. Cuando el examen reveló una lesión no varicosa con signos de hemorragia, se sometió a los pacientes a tratamiento endoscópico local. Los pacientes con úlcera péptica recibieron infusión continua de omeprazol endovenoso las primeras 72 horas, seguida de administración oral.

La estrategia transfusional restrictiva utilizó un umbral transfusional de hemoglobina menor a $7 \mathrm{~g} / \mathrm{dl}$ ( $\mathrm{n}=444$ pacientes), mientras que la estrategia liberal utilizó un umbral de hemoglobina de $9 \mathrm{~g} / \mathrm{dl}$ ( $\mathrm{n}=445$ pacientes). Se permitieron transfusiones con valores mayores de hemoglobina en casos de anemia sintomática, necesidad de cirugía o sangrado masivo.

\section{Medición de resultados}

El resultado principal a evaluar fue la mortalidad por cualquier causa en los primeros 45 días. Resultados secundarios fueron la frecuencia de extensión del sangrado (definida como hematemesis o melena con inestabilidad hemodinámica o descenso de la hemoglobina de $2 \mathrm{~g} / \mathrm{dl}$ o más en seis horas) y de complicaciones intrahospitalarias. El análisis se realizó por intención de tratar.

\section{Resultados principales}

La concentración de hemoglobina al ingreso fue similar en ambos grupos. La concentración mínima de hemoglobina en las primeras 24 horas fue menor en el grupo de estrategia restrictiva. La concentración de hemoglobina a 45 días fue similar en ambos grupos. El 51\% $(n=225)$ de los pacientes en el grupo de estrategia restrictiva y $14 \%(n=61)$ en el grupo liberal, no recibieron transfusiones $(P<0,001)$.

La mortalidad a 45 días fue significativamente menor en el grupo restrictivo: $5 \%(n=23)$ vs $9 \%(n=41)$ (Hazard ratio 0,55 ; IC95\% 0,33 a 0,92). Tanto la extensión del sangrado (10\% vs $16 \%$; HR 0,68; IC $95 \% 0,47$ a 0,98$)$, como la necesidad de cirugía de emergencia ( $2 \%$ vs. $6 \%, p=0,04)$ fueron menos frecuentes en el grupo restrictivo. Hubo también en este grupo menor tasa de eventos adversos ( $40 \%$ vs. $48 \%, p=0,02)$ y menor duración de la internación.

Entre los pacientes con cirrosis, tanto la mortalidad como la tasa de resangrado fueron menores en los pacientes del grupo restrictivo con clasificación Child Pugh A-B (HR para mortalidad 0,30; IC95\% 0,11 a 0,85), pero no en el subgrupo con cirrosis y clase $C$ de Child Pugh (HR para mortalidad 1,04; IC $95 \%$ 0,45 a 2,37 ). El resangrado por várices y requerimiento de terapia de rescate también fue menor.

Las reacciones transfusionales y eventos cardiovasculares, en especial el edema de pulmón, fueron más frecuentes en el grupo de estrategia liberal.

Se violó el protocolo en 39 pacientes del grupo restrictivo y 15 del grupo liberal.

\section{Conclusiones}

En comparación con la estrategia liberal, la estrategia restrictiva mejora significativamente los resultados en pacientes con SGAA.

Fuente de financiamiento: reporta no tener soporte comercial ni conflictos de interés.

\section{Comentario}

EI SGAA sigue siendo una de las complicaciones más graves del aparato digestivo y representa entre el 1 y el 3,5\% de las internaciones en servicios de emergencia. Se asocia con elevada morbimortalidad e indicación de transfusiones de glóbulos rojos, siendo discutida la estrategia transfusional más segura y eficaz.

Este artículo reporta una importante mejoría en resultados clínicamente relevantes al utilizar una estrategia restrictiva en comparación con otra liberal. Los hallazgos presentados apoyan los reportados en estudios observacionales así como las recomendaciones de expertos de transfundir con un umbral de hemoglobina de $7 \mathrm{~g} / \mathrm{dl}$ a los pacientes con hemorragia digestiva ${ }^{1-2}$.

Los resultados fueron obtenidos a partir de una muestra relativamente grande de pacientes lo cual aporta fortaleza a los mismos. Sin embargo, llama la atención la alta tasa de violaciones al protocolo, siendo mayor en el grupo restrictivo, sin encon- trarse una explicación del motivo o la evolución de estos pacientes. No se reportan tampoco datos de gran relevancia como edad, sexo o frecuencia de factores de riesgo o comorbilidades asociadas.

Dada la naturaleza de la intervención, la falta de cegamiento de la misma puede introducir sesgos importantes.

\section{Conclusiones de comentador}

En los pacientes con SGAA no masivo, el umbral para transfundir glóbulos rojos de $7 \mathrm{~g} / \mathrm{dl}$ de hemoglobina mejora los resultados, tanto de mortalidad como de complicaciones. Sin embargo, además de los niveles de hemoglobina, deben considerarse cuidadosamente factores clínicos como el estado hemodinámico, edad o comorbilidades, al elegir la estrategia transfusional apropiada.

\section{Tomas Caccavo [ Servicio de Clínica Médica del Hospital Italiano de Buenos Aires. tomas.caccavo@ @ospitalitaliano.org.ar ]}

Caccavo T. En el sangrado gastrointestinal alto agudo es mejor una estrategia transfusional restrictiva. Evid Act Pract Ambul. 2014:17(4).Oct-Dic. 123. Comentado de: Villanueva C, Colomo A, Bosch A, et al. Transusion strategie for acute upper gastrointestinal bleeding. N Engl J Med 2013; 368: 11-21. PMID 23281973.

\section{Referencias}

1. Barkun AN, Bardou M, Kuipers EJ, et al. International consensus recommendations on the management of patients with nonvariceal upper gastrointestinal bleeding. Ann Intern Med 2010; 152: 101-113.

2. Hearnshaw SA, Logan A, Palmer KR, et al. Outcomes following early red blood cell transfusion in acute upper gastrointestinal bleeding. Aliment Pharmacol Ther 2010; 32: 215-224. 